\title{
50 años de profesionalización de la antropología ecuatoriana: temas, derroteros y perspectivas
}

\author{
Fernando García
}

Carolina Páez²

'FLACSO Ecuador, Departamento de Antropología, Historia y Humanidades, Ecuador ${ }^{2}$ Pontificia Universidad Católica del Ecuador, Facultad de Ciencias Humanas, Ecuador

\section{Resumen}

Desde la década de 1940 las ciencias sociales ecuatorianas fueron testigo de varios esfuerzos realizados en miras a institucionalizar la enseñanza de la antropología en Ecuador. Este intento se materializó de manera definitiva en el año 1971 con la creación del primer departamento de antropología, adscrito a la Pontificia Universidad Católica del Ecuador (PUCE). En el camino recorrido es notorio la influencia de las diversas "antropologías del mundo". Hacia finales del siglo XX y comienzos del presente siglo se crearon otras carreras de pregrado y de posgrado, con lo cual el enfoque de la formación en antropología, los campos de acción y temas de interés se diversificaron exponencialmente. A cincuenta años de su profesionalización, en este artículo, exploramos los logros alcanzados, los derroteros transitados y los retos a los que se enfrenta la antropología en Ecuador. Palabras clave: antropología Ecuador, antropologías latinoamericanas, antropologías propias, identidades. 


\title{
50 anos de profissionalização da antropologia equatoriana: \\ temas, rumos e perspectivas
}

\section{Resumo}

Desde a década de 1940, as ciências sociais equatorianas têm testemunhado diversos esforços realizados para institucionalizar o ensino da antropologia no Equador. Essa tentativa foi finalmente concretizada em 1971 com a criação do primeiro departamento de antropologia, vinculado à Pontifícia Universidade Católica do Equador (PUCE). Ao longo do caminho percorrido, é notória a influência das várias "antropologias do mundo". No final do século 20 e início deste século, outras carreiras de graduação e pós-graduação foram criadas, com as quais o foco da formação em antropologia, os campos de atuação e os temas de interesse se diversificaram exponencialmente. Cinqüenta anos após sua profissionalização, neste artigo exploramos as conquistas, os caminhos percorridos e os desafios que a antropologia enfrenta no Equador.

Palavras-chave: Antropologia do Equador, antropologias latino-americanas, antropologias próprias, identidades.

\section{0 years of professionalization of Ecuadorian anthropology: themes, directions, and perspectives}

\begin{abstract}
Since the 1940s, the Ecuadorian social sciences have witnessed various efforts made to institutionalize the teaching of anthropology in Ecuador. This attempt was finally materialized in 1971 with the creation of the first department of anthropology, attached to the Pontifical Catholic University of Ecuador (PUCE). Along the way traveled, the influence of the various "anthropologies of the world" is notorious. Towards the end of the 2oth century and the beginning of this century, other undergraduate and graduate careers were created, with which the focus of training in anthropology, the fields of action and topics of interest diversified exponentially. Fifty years after its professionalization, in this article, we explore the achievements, the paths traveled and the challenges that anthropology faces in Ecuador.
\end{abstract}

Keywords: Ecuadorian anthropology, Latin American anthropologies, our own anthropology, Identities. 


\section{Introducción}

En el año 2019, los dos autores de este artículo, nos sumamos a la iniciativa de la Asociación Latinoamericana de Antropología (ALA), de crear el catálogo de tesis de antropología social de los distintos países de la región. Este esfuerzo conjunto abrió la posibilidad de profundizar la mirada y proponer preguntas sobre la producción antropológica en sus diferentes fases formativas. Así, iniciamos nuestra exploración sobre la identidad de la antropología en Ecuador a través de la indagación de los principales temas abordados por las tesis tanto de grado como de posgrado, con el fin de identificar la variación o permanencia de temáticas y hasta qué punto ésta permanencia o variación podría vincularse, por un lado, a cambios generacionales, y por otro a los programas de enseñanza. Esta exploración entra en sintonía con la conmemoración de los cincuenta años de profesionalización de la disciplina en el país.

El principal cuerpo de información se encuentra en el catálogo de tesis de antropología producidas en las distintas carreras ofertadas en el país en el período 1977-2019. Las tesis de grado (506) y de posgrado (343) se organizaron de acuerdo con la universidad que ofertaba el programa, el tipo de programa y la generación a la que pertenecen. Sobre esta información realizamos un análisis de contenido sobre los títulos de las tesis de acuerdo con la forma de organización planteada, es decir, a la titulación ofertada, a la universidad y a la década en la que fue producida. A través del análisis de contenido se buscaba identificar la recurrencia de categorías, para ello se eliminaron pronombres, adverbios, conjunciones y preposiciones. Para visualizar la recurrencia de categorías en los títulos de las tesis utilizamos la estrategia de nubes de palabras (words clouds). Las categorías más recurrentes se ubican en el centro de los gráficos y el tamaño de la palabra representa la recurrencia en relación a otras categorías de menor uso, las mismas que se ubican en los bordes y son de menor tamaño. Posteriormente, utilizamos listados cruzados para comparar la recurrencia de estas categorías entre titulación ofertada, universidad y década.

Para profundizar nuestro análisis sobre la identidad de nuestra antropología, recurrimos a la recolección de información primaria a través de un grupo focal con miembros de las primeras generaciones de antropólogos y antropólogas formadas en el país. En medio de la pandemia generada por el COVID 19 y las medidas de aislamiento y confinamiento aplicadas en Ecuador, recurrimos a una plataforma digital para la realización de esta actividad. Los temas principales giraron en torno a la experiencia universitaria y a la experiencia laboral. Con el fin de promover el proceso de evocación de los años universitarios, recurrimos a la técnica de foto elicitación (Harper 2002; Vivienne y Burgess, 2013).

En base a esta información proponemos que, si bien en las investigaciones fruto del proceso formativo se puede observar la multifacética "naturaleza" de la antropología, existen unos núcleos temáticos bien definidos tanto en la investigación formativa como una particular característica del quehacer antropológico. El eje que atraviesa estos núcleos temáticos es la pregunta por la identidad en sus diversas manifestaciones.

A continuación, presentamos los principales resultados de nuestra exploración. En primer lugar, hacemos un recuento de la institucionalización de la formación antropológica y su diversificación. En segundo lugar, describimos las tesis de antropología, sus temas principales y los derroteros de investigación incursionadas en las distintas universidades y generaciones. Por último, discutimos nuestros resultados en relación con los retos que enfrentan las antropologías latinoamericanas y la ecuatoriana en específico.

1 Se trata del Catálogo Latinoamericano de Tesis de Antropología Social, que forma parte de un grupo de investigación de la Asociación Latinoamericana de Antropología (ALA), constituida de hecho en 1990 y legalizada en el año 2018. En la actualidad cuenta con información de 9 países, ha catalogado 21.000 tesis en el período de 75 años (1945-2020). 


\section{Trayectorias formativas de la antropología ecuatoriana}

Desde la década de 1940 Ecuador fue testigo de varios esfuerzos realizados en miras a institucionalizar la antropología. En el marco general de las políticas indigenistas y desarrollistas, se crearon espacios de diálogo y acción entre profesionales de la antropología que se habían formado en el extranjero y en el país, a través de instituciones como el Museo de Arqueología Nacional de la Universidad Central del Ecuador (1925), reabierto en 1959 con un enfoque más etnográfico, el Instituto Indigenista Ecuatoriano (1943), el Instituto Ecuatoriano de Folklore (1950), el Instituto Ecuatoriano de Antropología y Geografía (1950), el Servicio Ambulante Rural de Extensión Cultural (SAREC), la Misión Andina del Ecuador (1954) (Prieto y Páez, 2017) y el Instituto Otavaleño de Antropología (1966). A pesar de la existencia de estas instituciones ninguna de ellas ofreció un espacio de formación de nuevos profesionales.

La antropología se configuró como una ciencia comprometida, que en sus inicios estuvo vinculada con el indigenismo, marcada por la influencia de la antropología mexicana y peruana (Barba, 2020; García, 2011; Martínez, 2008; Moreno, 2006; Prieto, 2010). Tanto Perú como México, junto con Brasil, van a ser los centros productores de pensamiento antropológico de la época, y sus políticas públicas respecto al tema indígena, se convirtieron en referentes regionales. Investigaciones recientes que han profundizado el análisis del proceso de formación del campo antropológico en el país identifican "al indigenismo como una manifestación intelectual que abarcó desde diversos registros el problema del indígena frente a la construcción del Estado nación” (Barba, 2020: 11). Sin embargo, en otro lugar (García, 2014), hemos propuesto que si bien Ecuador contaba con intelectuales indigenistas, no existió una política de Estado, institucionalidad y recursos permanentes para hacer de la antropología una disciplina instaurada de forma definitiva en el país.

La profesionalización de la disciplina se consolidó con la creación del primer departamento de antropología en la Pontificia Universidad Católica del Ecuador en el año 1971. En la experiencia formativa en Ecuador se priorizaron los temas campesinos e indígenas. En el contexto de las transformaciones relacionadas a las reformas agrarias de 1964 y 1973 y de las propuestas de la antropología marxista, el principal núcleo temático de las primeras generaciones de tesis de antropología fueron los estudios campesinos, enfocados en los cambios sociopolíticos y económicos. Igualmente, dirigida por el ímpetu de Marco Vinicio Rueda, otra de las líneas de indagación bien definida fueron los temas religiosos, la cosmovisión, la fiesta, en definitiva, el mundo simbólico (García, 2011; Martínez, 2008; Moreno, 2006). En las imágenes que siguen, que son mapas conceptuales sobre los temas de investigación de tesis, se muestran la tesis de la PUCE en dos períodos,1977-1989 y 1990-1999. (Ver imagen $1 \mathrm{y}$ 2). 


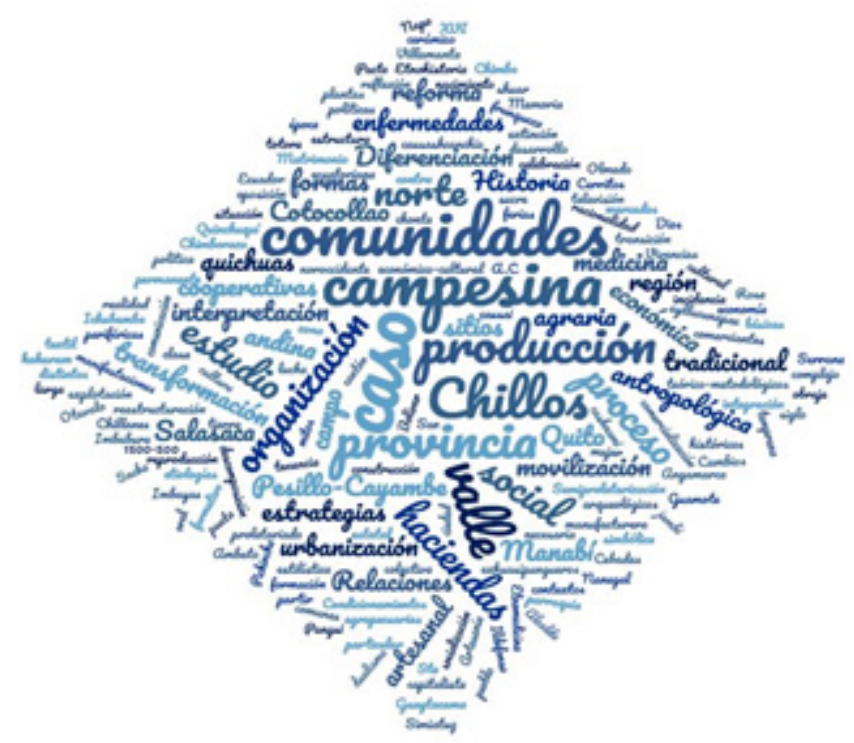

Fuente: Listado de tesis - Laboratorio de antropología PUCE 1977 - 2019. Elaboración propia

Como se puede observar en la Imagen 1, los núcleos temáticos preponderantes durante el período 1977 - 1989, cuando la PUCE era el único centro de educación superior que ofertaba la carrera de antropología, eran los análisis sobre el mundo campesino, las comunidades, las haciendas, la producción. Esta tendencia, como se describió líneas más arriba, estaba vinculada a las reformas agrarias y a las transformaciones correlacionadas en torno a la tierra, la economía y la política. Asimismo, la concentración en estos temas estuvo vinculada a los esfuerzos por implementar una escuela de campo que permitiera a la antropología estar en contacto cercano con los problemas que enfrentaban los actores sociales, en especial los pueblos indígenas, para así acompañar a la transformación de las formas de explotación a las que habían estado sujetas.

Imagen 2. Tesis de antropología PUCE 1990 - 1999

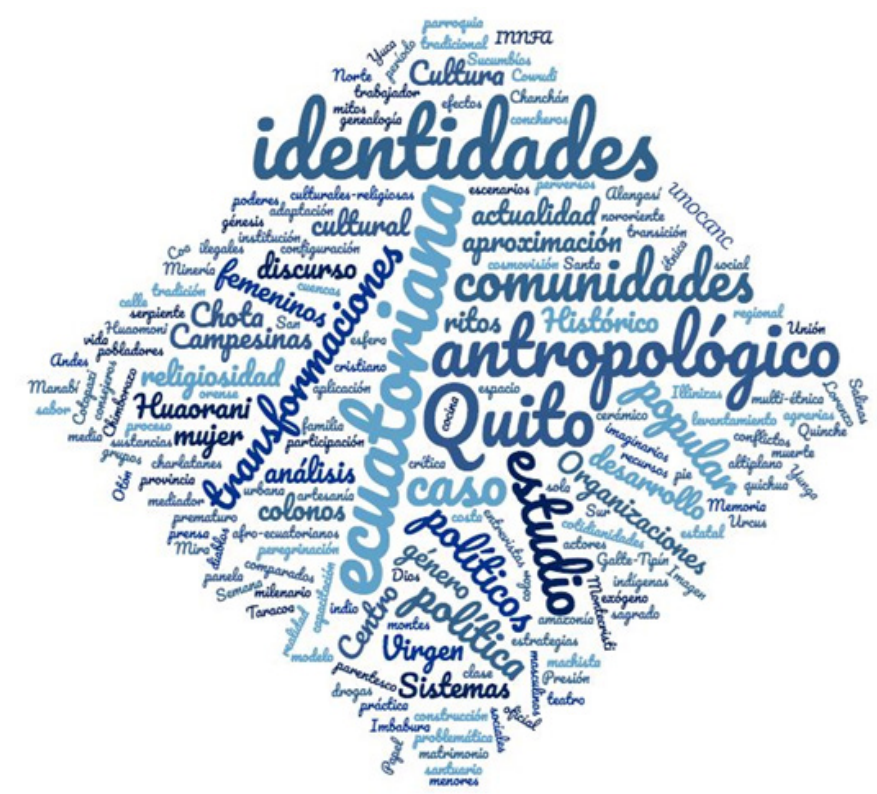

Fuente: Listado de tesis - Laboratorio de antropología PUCE 1977 - 2019. Elaboración propia 
Por otro lado, en la Imagen 2 se puede observar que los temas campesinos si bien no desaparecen van compartiendo espacio con temas de religiosidad, ritualidad y género, mientras que el tema de las identidades se convierte en preponderante.

El estudio de los pueblos indígenas amazónicos fue tomando forma a partir de la década de 1970 por el interés de dos actores: académicos extranjeros que realizaron sus investigaciones de campo en el país y la incidencia de órdenes religiosas como la de los salesianos y capuchinos en el contexto de los procesos de colonización de la Amazonía. Los salesianos han jugado un rol preponderante en la producción antropológica, no solo con la oferta académica de las carreras de peritaje antropológico (en la que se reportan graduados en el período 1990 - 2002) y de antropología aplicada que se creó en 1987 en la Universidad Politécnica Salesiana, por la influencia de Juan Botasso (ver imagen 3), sino también con las publicaciones a través de la editorial Abya-Yala (1975). De acuerdo con Martínez (2008), el interés de la antropología por temas indígenas, no solo se da en el contexto de las reformas agrarias, también está anclado al peso político del movimiento indígena en Ecuador desde la década de los 80.

Imagen 3. Tesis de antropología aplicada UPS 1990 - 1999

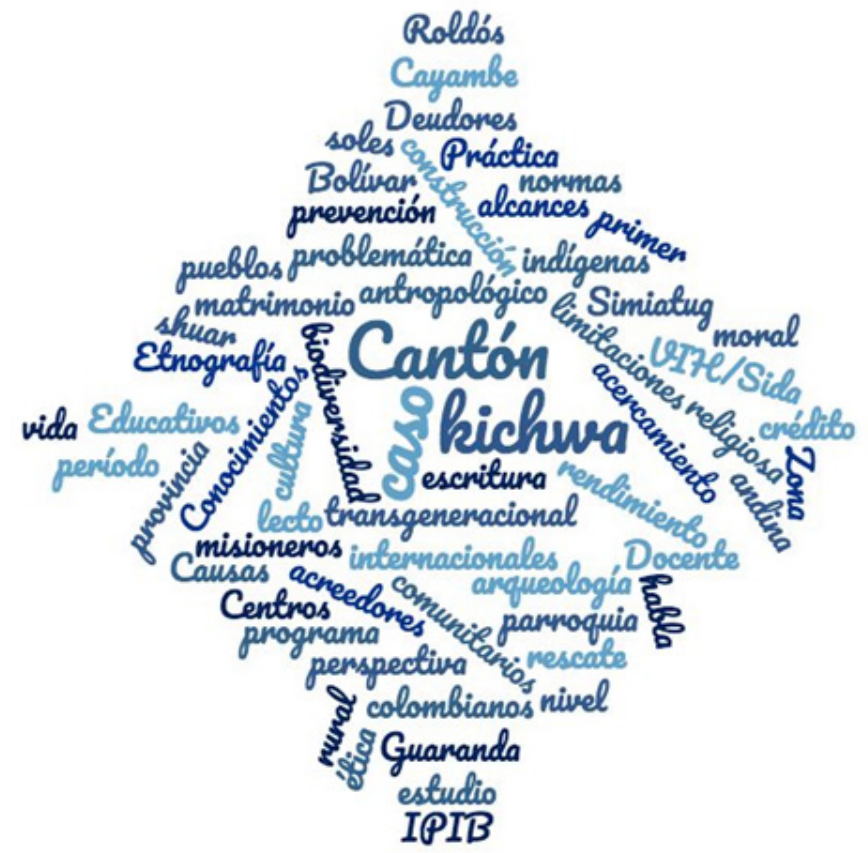

Fuente: Listado de tesis - Laboratorio de antropología PUCE 1977 - 2019. Elaboración propia

Hacia finales del siglo 20 se crea otra carrera, esta vez de posgrado, en la Facultad Latinoamericana de Ciencias Sociales, con lo cual el enfoque de la formación en antropología, los campos de acción y temas de interés se diversificaron exponencialmente. Con la creación de la maestría en antropología en la FLACSO a inicios de los años 1990, la apertura de la antropología ecuatoriana al diálogo y la influencia de antropologías extranjeras configura un campo vibrante y problemático, por el sentido "cosmopolita" que caracteriza a esta institución de educación superior, pero al mismo tiempo por su "dependencia" a centros académicos "fuertes" (Martínez, 2008) (Ver imagen 4). 


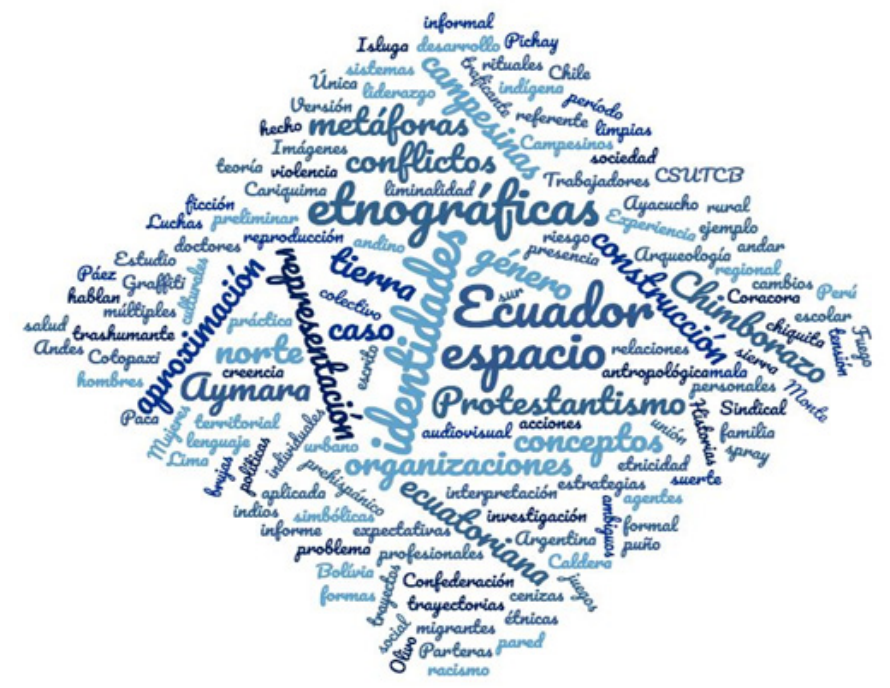

Fuente: Listado de tesis - Laboratorio de antropología PUCE 1977 - 2019. Elaboración propia

Entre los temas abordados por las tesis producidas en FLACSO en el período 1993-1996, se ha podido identificar que la indagación por identidades ocupa un lugar central. De igual manera, las investigaciones en torno a las representaciones y las construcciones sociales se posicionan de manera importante; los temas étnicos y religiosos se mantienen en estas tesis.

Si bien existieron otros proyectos educativos a nivel de posgrado, estos fueron más eventuales; tal es el caso de la maestría en antropología de la Universidad del Azuay, la cual tuvo una vigencia de dos años (1991-1992) (Ver imagen 5). Sus graduados se concentraron en los temas clásicos de las primeras generaciones, tales como los estudios sobre el campesinado, la configuración comunitaria y los asuntos de género.

Imagen 5. Tesis de maestría de antropología. Universidad del Azuay 1991 - 1992

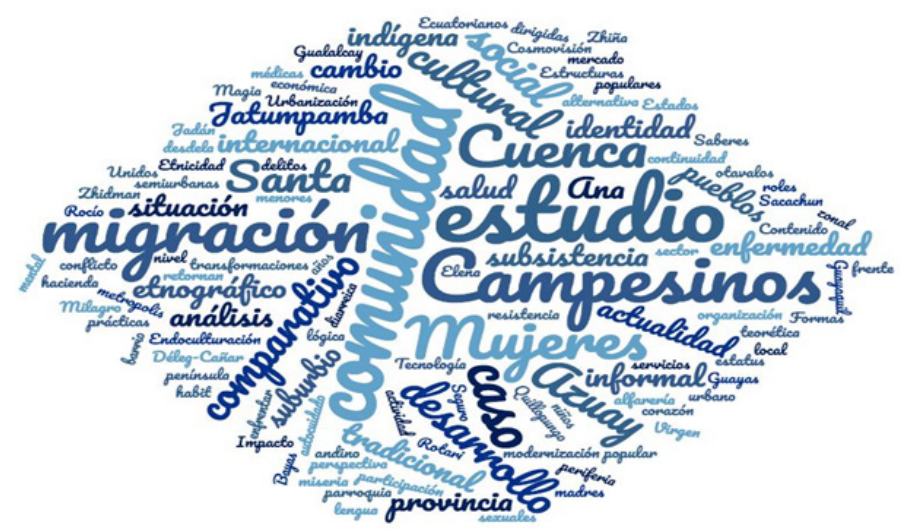

Fuente: Listado de tesis - Laboratorio de antropología PUCE 1977 - 2019. Elaboración propia. 
La creación de la maestría en antropología visual en la FLACSO a partir del año 2008, de la maestría en antropología de lo contemporáneo en la Universidad de Cuenca del 2018 al 2019, otra maestría en la Universidad Politécnica Salesiana del 2009 al 2014 y el grado en artes liberales concentrado en antropología en la Universidad San Francisco de Quito a partir del año 2014, diversificó los enfoques, temas y abordajes de la antropología, a pesar de mantener como uno de sus ejes las preguntas por la identidad, asunto al que volveremos más adelante (Ver imágenes 6, 7, 8 y 9).

Imagen 6. Tesis de maestría de antropología visual FLACSO 2008 - 2019

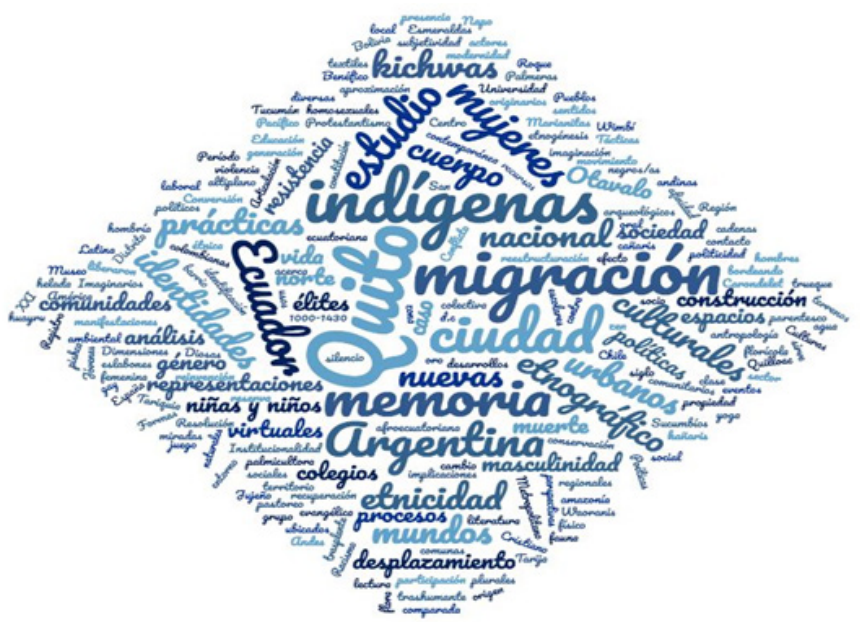

Fuente: Listado de tesis - Laboratorio de antropología PUCE 1977 - 2019. Elaboración propia

De manera específica, los trabajos de titulación de la maestría en antropología visual han posicionado temas como la aproximación al fenómeno urbano y a la migración. Han mantenido su espacio las indagaciones sobre la etnicidad, las representaciones y los asuntos de género. De manera similar a los otros programas de estudio, la investigación sobre las identidades ha mantenido un lugar relativamente importante.

Imagen 7. Tesis de maestría de antropología Universidad de Cuenca 2018 - 2019

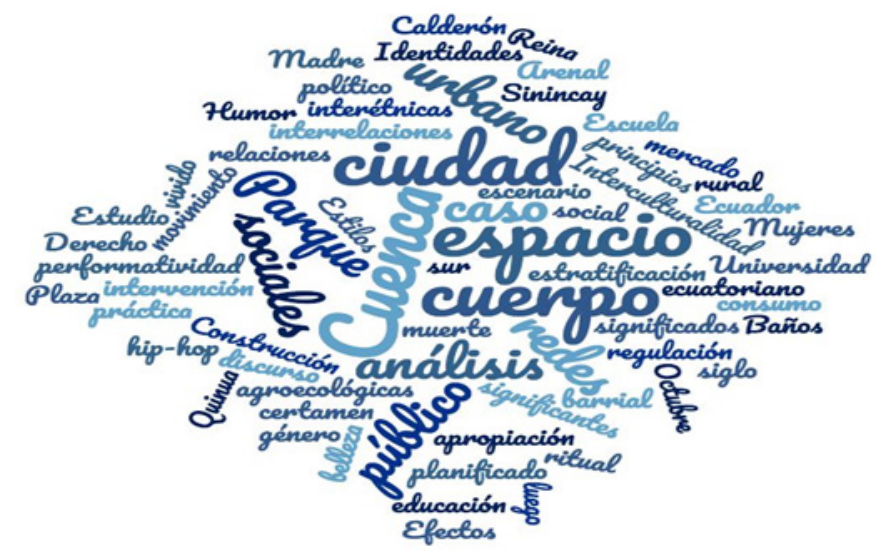

Fuente: Listado de tesis - Laboratorio de antropología PUCE 1977 - 2019. Elaboración propia 


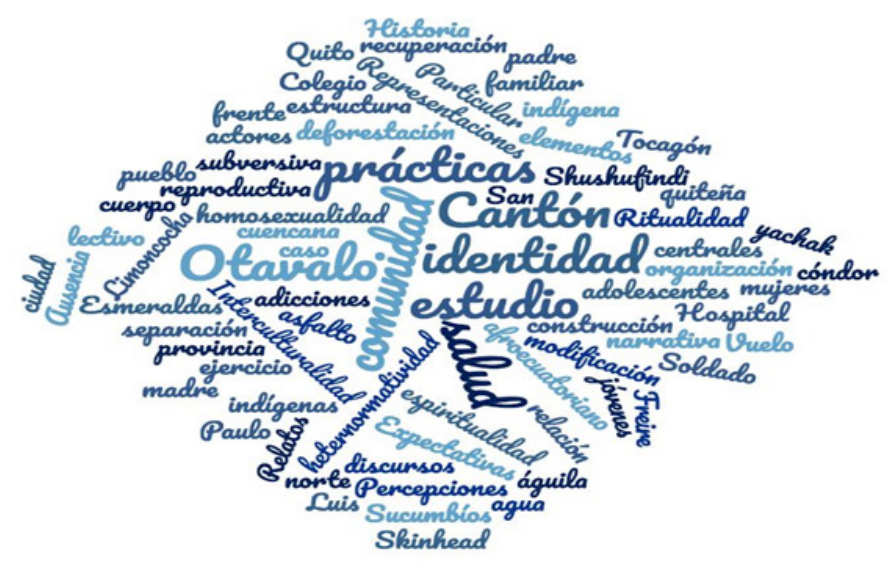

Fuente: Listado de tesis - Laboratorio de antropología PUCE 1977 - 2019. Elaboración propia

Por su parte, en las investigaciones de maestría de la Universidad de Cuenca y de la UPS, que han tenido una temporalidad menor a las maestrías ofertadas por FLACSO, han preponderado los temas urbanos, la ciudad, los espacios, el cuerpo y la performatividad, en el primer caso. En el segundo caso, los temas se han enfocado principalmente en la comunidad, la antropología aplicada y la identidad.

Finalmente, en el caso de las tesis de grado de la USFQ, durante el período 2014 - 2019 también han abordado temas vinculados con la identidad, relacionados de manera especial con la exploración de los asuntos de género y diversidades sexo genéricas, así como con la edad.

Imagen 9. Tesis de antropología de USFQ 2014 - 2019

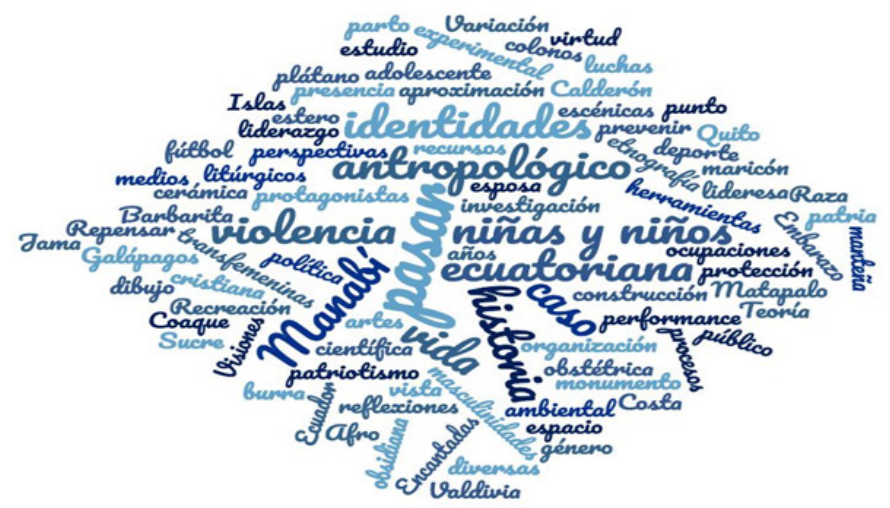

Fuente: Listado de tesis - Laboratorio de antropología PUCE 1977 - 2019. Elaboración propia

El desarrollo de la antropología en Ecuador se ha concentrado en la antropología social y la arqueología, otros dominios como la lingüística ha tenido su desarrollo en la carrera que ofrece la Pontificia Universidad Católica del Ecuador. Por su lado la antropología física no ha contado con un espacio de formación.

Otro hecho significativo es que las opciones de formación se han concentrado en la capital del país, a excepción de la escuela de arqueología que funciona en Guayaquil en la Escuela Superior Politécnica del Litoral (ESPOL). Este hecho es una limitación para la formación de profesionales en provincias, de alguna manera respondida con la carrera de la Universidad Politécnica Salesiana que se oferta en modalidad virtual. En la siguiente sección miramos con más detalle los temas de interés en la investigación formativa. 
Finalmente, con excepción de la carrera de arqueología de la ESPOL y de la maestría de la Universidad de Cuenca, las opciones de formación para antropólogos/as han sido ofrecidas en universidades particulares o internacionales, por lo que es notable la ausencia de la universidad públicas en este campo.

\section{La identidad antropológica}

En la sección anterior describimos la trayectoria de la formación académica de la antropología en el país. En las décadas de 1970 y 1980 el único programa ofertado fue el de grado en la PUCE. Las otras universidades que mantienen una permanencia en el tiempo en su oferta académica son el pregrado de la UPS y el posgrado de la Flacso Ecuador, mientras que las otras ofertas han sido episódicas (maestrías en la UDA, la UCuenca) o mucho más recientes (grado en la USFQ) o que cerraron y se volvieron a abrir (arqueología en la ESPOL).

Basados en esta característica, Bustamante y Carrión (2021) periodizan la producción de tesis en tres momentos: de 1977 a 1985, de 1985 a 2005 y de 2005 a 2019. En el primer momento, el tema principal de investigación estuvo relacionado con la cuestión indígena (64.1\%), y de manera especial con temas agrarios e históricos. De acuerdo con Pérez (2020: 5), "La antropología se erigía... como una disciplina útil para el acercamiento y el análisis de los discursos y prácticas que se originan y se asimilan en el seno de las sociedades con relación al desarrollo y con los sujetos de que este desarrollo conforma".

Dentro de los temas emergentes abordados en los trabajos de titulación a mediados de la década de los ochenta y principios de la década de los noventa, es posible identificar un interés por la vinculación con los temas de género y étnicos (Poeschel 1985), la televisión (Rebolledo, 1985), el pueblo afroecuatoriano (Escobar, 1988), las dinámicas socioculturales propias del consumo y tráfico de drogas (Andrade, 1992), los conflictos socio ambientales generados por el extractivismo (Murillo, 1992), las culturas juveniles (Ensignia, 1993) y la alimentación (Carrillo, 1995).

A pesar de la permanencia de los temas indígenas, la incursión en otras temáticas empieza a tomar fuerza a mediados de los años ochenta, intensificándose en la década de 1990, dentro de lo que se ha denominado como el "giro cultural", las preguntas vinculadas a las desigualdades y la crisis de los paradigmas en las ciencias sociales (Reygadas, 2007). Es decir, hay una diversificación de los temas de investigación y de la oferta académica en grado y posgrado. Según Rocío Pérez $(2020,4)$, en el análisis comparativo que realiza sobre el sujeto antropológico en Ecuador y Bolivia, es en este momento, los años ochenta y noventa que

la antropología recuperaría su interés en la alteridad y la diferencia (desigualdades socioculturales) aunque extrapolando esta preocupación hacia el conjunto de la sociedad ecuatoriana. De este modo, la disciplina comenzó a abrirse a una interdisciplinariedad que abogaba por unos marcos teórico-metodológicos más flexibles, experimentales y variados que se adecuaran a una realidad compleja.

Si bien estos temas emergentes dan cuenta de la diversificación en los intereses de investigación de los y las estudiantes, dentro del segundo momento (1985-2005) identificado por Bustamante y Carrión (2021), los temas más recurrentes en las tesis se enfocaron en la religiosidad, los rituales y los mitos. Asimismo, se concentraron de manera importante en temas de economía y políticas públicas.

En el tercer momento (2005-2019) aparecen de manera importante temas vinculados al arte, la ciudad, el género y la ecología. Los estudios de mujeres y de género se intensifican y diversifican hacia finales de los años noventa, sin embargo, su incursión obedece más bien al interés de las y los estudiantes antes que a la incorporación en el pensum de la antropología de género, especialmente en la PUCE (Prieto, 2007). En el nuevo milenio, las diversidades sexo genéricas y los estudios de masculinidades irrumpen en el escenario de las tesis en sus distintos niveles, así como la ampliación hacia otros grupos definidos por la edad (niñez, juventud, 
y tercera edad). A su vez, la antropología urbana se configura como una línea bien establecida enfocada en los espacios, los imaginarios y las formas diversas de habitar y construir la ciudad.

Dentro de los temas emergentes de la última década vale la pena mencionar el estudio sobre las discapacidades, que abarca desde las prácticas y representaciones de las diferencias en el entorno escolar (Rojas, 2011), la inclusión laboral (Pico, 2014), visualidad (Idrovo, 2014; Montellano, 2011), y lenguaje de señas (Acosta, 2011; Bossano, 2019).

Los análisis recientes sobre el período que abarca el último momento de producción de tesis ubican a la noción del Buen Vivir como un factor importante en la identidad de la antropología ecuatoriana (Pérez, 2020; Piñeiro, 2020). Este paradigma alternativo al desarrollo -junto al Sumak Qamaña en Bolivia-, "atraería (de diferentes formas y aportaciones) a la antropología, incluso a la antropología postdesarrollista más crítica quien vio una potencialidad y una innovación conceptual que no se veía desde la teoría de la dependencia latinoamericana en las décadas de los 60, 70, y 80 del siglo XX (Hidalgo y Cubillo, 2017)" (Pérez, 2020: 8).

De acuerdo con Bustamante y Carrión (2021), vistas en conjunto los cuatro principales temas abordados por las tesis de antropología sociocultural se remiten a: a) política y políticas públicas, b) religiosidad, ritualidad y simbología, c) esstudios urbanos, d) género. A su vez, la concentración en distintas identidades puede identificarse acorde a la adscripción étnico racial, el campesinado, las actividades económicas personificadas en la identidad de las ocupaciones y las identidades configuradas por el género, tal como se muestra en la tabla 1:

Tabla 1: Investigación formativa según identidades

\begin{tabular}{|c|c|}
\hline Pueblos y nacionalidades & $23.3 \%$ \\
\hline Campesinos no indígenas & $14.2 \%$ \\
\hline Grupos ocupacionales & $8.9 \%$ \\
\hline Identidades de género & $8.4 \%$ \\
\hline
\end{tabular}

Fuente: Bustamante y Carrión 2021.

En la sección anterior señalamos la creación de los distintos programas académicos de grado y posgrado tanto en antropología como en arqueología. Se ha podido observar que si bien han existido programas en otras provincias, especialmente de la región Sierra, los programas con mayor permanencia en el tiempo se concentran en Quito, la ciudad capital (Piñeiro, 2020). Bustamante y Carrión (2021) identifican que no solo la oferta se concentra en Quito, la investigación tiene una marcada inclinación a realizarse en la provincia de Pichincha, junto a otras provincias de la Sierra Norte y Centro. Le siguen en concentración de tesis las realizadas en la Amazonía y en la región Costa. No hay que olvidar el surgimiento del movimiento indígena ecuatoriano, concentrado en la Sierra y Amazonia, va a constituir un elemento atractivo para las investigaciones inspiradas en temas étnicos y culturales. Un porcentaje importante de las tesis se realiza en el extranjero; los autores identifican que la mayoría de estas investigaciones son sobre Brasil, en el marco del programa de grado en antropología aplicada de la UPS, que como se señaló más arriba se oferta en modalidad virtual (Tabla 2).

Tabla 2: Investigación formativa según región

\begin{tabular}{|c|c|}
\hline Región & Porcentaje \\
\hline Sierra & $52.9 \%$ \\
\hline Amazonía & $8.3 \%$ \\
\hline Costa & $8.2 \%$ \\
\hline Insular & Sin información \\
\hline Extranjero & $14.1 \%$ \\
\hline
\end{tabular}


Si bien existe esta concentración, en términos generales, al igual que en otros países de la región, la concentración en temas identitarios ha ido de la mano con el esfuerzo por "visibilizar problemáticas sociopolíticas en las que los antropólogos han interactuado con otros agentes" (Piñeiro, 2020: 4).

\section{¿Quiénes somos? ¿Qué hacemos?}

En las secciones anteriores describimos la creación de los programas académicos de grado y posgrado en antropología y arqueología en el país, desde su institucionalización en 1971. Asimismo, describimos los núcleos temáticos de la investigación formativa plasmada en las tesis a lo largo de los 50 años de profesionalización de la antropología ecuatoriana. En esta sección nos adentramos en las percepciones de los y las profesionales del ramo. En primer lugar, contextualizamos esta experiencia en términos comparativos con la institucionalización de la profesión en otros países de la región. En segundo lugar, abordamos los repertorios que emergen sobre lo qué es la antropología y lo que hace.

Análisis recientes sobre las antropologías andinas han señalado que la disciplina se institucionaliza, en términos comparativos, tardíamente en Ecuador. Por ejemplo, Gómez (2020) señala que en Perú y Colombia estos procesos se dan a inicios de la década de 1940. En el caso peruano, si bien se crea la carrera en la Universidad Mayor de San Marcos (1945), un ejercicio precedente fue la sección de Historia y Antropología en la Universidad San Antonio de Abad, en el Cuzco. La creación de la carrera se dio a la par a la creación del Instituto Francés de Estudios Andinos (1948) y del Instituto de Estudios Peruanos (1964) y más tarde la creación del departamento en la Pontificia Universidad Católica del Perú (1969). Al momento, en Perú la oferta académica cubre gran parte del territorio, tan solo 3 de las 10 carreras ofertadas se ubican en Lima (Gómez, 2020: 7).

En el caso colombiano, el proceso de institucionalización se solidifica en 1941 con la creación del Instituto Etnológico Nacional y la apertura del programa de antropología en la Universidad de los Andes (1963), de la Universidad Nacional (1966). Les siguieron las universidades de Antioquia (1966) y la del Cauca (1970). En la actualidad la oferta de grado cuenta con 15 programas y alrededor de 6 de posgrados (Gómez, 2020: 7).

Al igual que en el resto de la región, la antropología ecuatoriana se caracteriza por su "vocación crítica" (Jimeno, 2007) y por el entretejimiento entre la práctica aplicada y la académica (Gómez, 2020; Piñeiro, 2020), en palabras de nuestros entrevistados:

La escuela de antropología se crea con un propósito muy aplicado. La decisión de Hernán Malo (rector de la PUCE en 1971) para crear una escuela de antropología, influida por su discurso de "ecuatorianizar la universidad". El departamento se crea como el significante de la ecuatorianización de la universidad más centrada en la problemática propia del país y menos interesada en lo cosmopolita de la ciencia o las carreras. Esa idea vino a ser retomada en el liderazgo de Alfonso Gortaire. Gortaire había creado el Instituto de Pastoral Latinoamericana, que funcionó en Quito. Era una escuela para formar misioneros posconciliares que trabajaran con los sectores populares. De una manera muy señalada con los sectores indígenas, junto con la Compañía de Jesús. Con una vocación aplicada. La creación del departamento tiene una impronta jesuita, la mitad de los alumnos vienen del filosofado San Gregorio (Diego Iturralde, entrevista 2021).

Se debe ubicar los procesos sociales con los que va la antropología, a qué se estaba respondiendo con esas diferentes tendencias o momentos que tanto en la PUCE y en la Salesiana por la influencia del padre Botasso, fue moviéndose en ese tipo de análisis para hacer la antropología aplicada (Martha Núñez, entrevista 2021)

Nuestra antropología tuvo como tema referencial el Estado, y los movimientos frente al estado, pero hemos podido romper, hay una producción que va más allá de la dinámica del Estado, de las pautas con las que nos formamos o las que todavía usamos para discutir (José Almeida, entrevista, 2021). 
Es desde esta vocación crítica y desde las difusas líneas entre la práctica académica y la aplicada que la antropología adquiere una identidad de servicio:

La antropología surge en el contacto con el otro. El lugar de la antropología está en la relación interétnica, en lo intergeneracional, es un aparato de traducción cultural para establecer diálogos equitativos entre los tradicionalmente desiguales. Ahí aprendí a contestar la pregunta: ¿para qué les ha servido la antropología a los diferentes grupos con los que he trabajado, como abogado y antropólogo?, le ha servido a los movimientos sociales, al movimiento indígena, a algunas instituciones públicas (Diego Iturralde, entrevista 2021).

Es interesante señalar que la acción de la antropología ha tenido a dos interlocutores fundamentales como son los movimientos sociales y el Estado con todo su aparato de leyes, normativas y política públicas. Es con ellos con los que se va a mantener una actitud de debate, confrontación y colaboración a través del tiempo.

Otra colega pone énfasis en la acción de la antropología de acompañar los procesos sociales, no solamente como meros testigos, sino como actores sociales participantes y comprometidos con los conflictos que ha atravesado la sociedad ecuatoriana a través del tiempo.

No todos manejan los elementos que nosotros manejamos. Otros profesionales, economistas, periodistas, pueden saber de antropología, pero no tienen los elementos. Esos elementos no están en la teoría. Tenemos que estar en esos lugares donde se discuten cosas que afectan a la gente, como la agenda 2030 o los ODM ¿Qué significa esas cosas? Entonces hemos estado en esos procesos de traducir, de acompañar. En la salida del Instituto Linguístico de Verano por ejemplo, que formamos el frente de defensa. Fuimos el único movimiento capaz de acompañar justamente porque no nos quedamos en el membrete del proletariado. (Martha Núñez, entrevista 2021)

Finalmente, la necesidad de entender las dinámicas sociales desde la visión de sus propios actores aparece como una característica de la práctica profesional de la antropología como ciencia social. De alguna manera se menciona también, el compromiso político de los antropólogos/as con los sectores sociales investigados.

Yo acudí a la antropología por necesidad. Yo estuve trabajando en los años 60 con sectores indígenas especialmente de Tungurahua y Chimborazo en procesos de reivindicación de tierras. Esos procesos fueron dolorosos e inclusive hubo un indígena asesinado, me partió el alma. Hasta dudé de Dios. Para sintetizar. La cuestión es que la reforma agraria fue adelante y en la zona donde yo trabajaba se pudo conseguir la adjudicación de 7 haciendas, con tensiones y todo pero se pudo conseguir. Ahora van a festejar 50 años de ese hecho. Cuando las tierras salieron y la gente se organizó en cooperativas. Pero debajo de las cooperativas y del IERAC, había las organizaciones que marcaban su propia forma en la que manejaban la tierra, se interrelacionaban, manejaban la producción. Ahí vi, mientras se daba esa confrontación terrible sobre el derecho a la tierra, que yo era alguien que podía colaborar con los pueblos indígenas de esas zonas. Cuando eso se logró, yo entendí que era urgente que se reivindique la dimensión de cómo era hacerlo desde sus propias visiones. Eso es lo que me llevó a la antropología (Eulalia Carrasco, entrevista 2021)

De acuerdo a los y las participantes de este grupo, en la formación profesional, el trabajo de campo es una marca constitutiva y una manifestación de su vocación crítica. Su versatilidad le permite acompañar no solo a otros actores sociales y profesionales, también al avance de derechos colectivos y procesos reivindicativos. Pese a las virtudes que se le atribuye a esta profesión, las preocupaciones de los y las participantes se concentraron en la precariedad de un ámbito laboral reducido, flexible y de poca regulación. Por ende, emerge la demanda de la organización gremial que no solo vele por los derechos laborales, sino que también se convierta en una voz pública.

A propósito de esta demanda cabe señalar que en el año 1981 se creó el Colegio de Antropólogos y Lingüistas de Pichincha (CALP), con el apoyo del Colegio de Etnólogos y Antropólogos Sociales (CEAS) de México. Estuvo conformado por antropólogos y lingüistas de la PUCE y mantuvo actividades hasta el año 2012. 


\section{Los retos hacia el futuro}

Se puede apreciar que el pensamiento antropológico ecuatoriano ha mantenido una relación muy estrecha con tres antropologías del sur, la mexicana sobre todo por los ecuatorianos/as formados en ese país, la peruana por ser el referente de los estudios andinos y la colombiana por la vecindad y por compartir características y problemáticas semejantes.

Paradójicamente se conoce muy poco de las otras antropologías del sur, en especial las latinoamericanas, nos referimos a las antropologías del cono sur, como por ejemplo la brasileña y la argentina. En especial la primera que actualmente constituye junto con la mexicana y andina el marco de referencia obligado de la antropología latinoamericana.

Desde esta perspectiva cobra especial importancia el surgimiento y consolidación de espacios de debate y discusión surgidos en los últimos años. Nos referimos a tres en especial, la Asociación Latinoamericana de Antropología (ALA) creada en 1990 en un congreso de la Asociación Brasilera de Antropología (ABA) en Florianópolis, que ha llevado adelante seis congresos y reúne al conjunto de gremios profesionales de la región.

Luego, la Red Latinoamericana de Antropología Jurídica (RELAJU) formada en 1997 en Quito, Ecuador, en el marco del $49^{\circ}$. Congreso de Americanistas. Hasta la fecha ha convocado a nueve congresos regionales. Finalmente, la Reunión de Antropología del Mercosur (RAM) que es un encuentro bianual que se realiza desde 1995, organizado por instituciones e investigadores de los países del MERCOSUR, en el año 2009 se realizó la octava reunión que prácticamente se convirtió en un evento latinoamericano por el número y el origen de sus participantes.

No dejaremos de mencionar el interés que muestran los colectivos nacionales de antropólogos/as por convocar periódicamente sus congresos nacionales, así el caso de Brasil, Argentina, Chile, Perú. Ecuador, Colombia, Costa Rica y México.

Acogemos una de las sugerencias de Krotz (2007) de estudiar las antropologías del sur haciéndolas visibles para aprender desde el sur y no sólo desde el norte. Es indispensable, como también lo señala Krotz, "utilizar la ciencia antropológica para estudiar la ciencia antropológica" (2007: 169), ya que solamente de esta manera podremos iniciar un gran ejercicio, propio también de la antropología, de comparación de cómo estamos construyendo y enseñando el pensamiento antropológico en cada uno de los países de la región.

Vale la pena mencionar, desde nuestra perspectiva, los principales aportes con que la antropología ecuatoriana ha contribuido a la antropología latinoamericana. En realidad, se concentran en tres temas, los estudios específicos de los denominados Andes del Norte, en contraste con lo que sucede con los Andes del Sur, los peruanos y bolivianos. El segundo tema tiene que ver con las contribuciones para entender la dinámica de los pueblos asentados en la alta Amazonia, que les hace diferentes a los ubicados en la parte baja de la gran cuenca amazónica. Por último, la antropología ecuatoriana ha participado en el gran debate de incorporar en la lucha social no solamente los elementos de clase, sino sobre todo los factores étnicos y culturales, que se han convertido con el tiempo en motivos de movilización política y económica.

Respecto a la participación como gremio profesional y como centros académicos de formación de pre y post grado en el contexto nacional, es importante mencionar su contribución en la discusión de la diversidad cultural y étnica al interior de las políticas públicas, así como el apoyo a la adopción del enfoque de derechos individuales y colectivos al interior de la sociedad ecuatoriana.

Otro reto importante es la necesidad de descentralizar la enseñanza de la antropología en Ecuador, cuatro de las cinco universidades que enseñan la carrera están en la capital del país, aunque el recurso de la enseñanza virtual puede cambiar esta realidad. 
Junto con el anterior se hace indispensable que las universidades públicas ofrezcan este tipo de formación, la excepción es la Universidad de Cuenca que ha ofrecido una maestría. No hay que olvidar un hecho histórico, fue en la Universidad Central de Quito que se iniciaron las primeras actividades académicas antropológicas con los cursos ofrecidos por Antonio Santiana de Etnología y Arqueología, en la Escuela de Pedagogía de la Facultad de Filosofía, Letras y Ciencias de la Educación, desde el año 1946 (Barba, 2020). Los y las profesionales de la antropología provienen no solamente

de los sitios donde se enseña la disciplina, sino también de todas partes del país. Hay un importante grupo de antropólogos/as indígenas y afroecuatorianos formados en los últimos 30 años, que han dinamizado la discusión y debate desde sus propias cosmovisiones, se hace necesario impulsar nuevas escuelas de formación que prioricen tanto la adscripción a universidades estatales como la apertura de nuevos centros en otras ciudades del país, en especial en la Costa y Amazonia.

Finalizamos haciendo nuestras las preguntas que se plantean las coordinadoras del dossier denominado Antropología sobre América Latina y el Caribe hoy: nuevos desafíos teóricos y metodológicos y las convertimos en retos hacia el futuro. ¿Cómo ha contribuido o puede contribuir la antropología y el conocimiento que produce a resolver los principales problemas nacionales y regionales en un contexto más global? ¿Cómo se acumulan los conocimientos antropológicos sobre el tema de la desigualdad, ya sean étnicos, raciales, religiosos, de género o de clase, son o deberían ser un referente para la construcción de sociedades más armoniosas y abiertas a la diversidad? ¿Qué paradigmas y metodologías ha desarrollado la antropología latinoamericana para comprender los problemas sociales que investigamos? ¿Cuál ha sido y puede ser el vínculo entre la Antropología y el Estado, especialmente en el contexto de la elaboración de políticas públicas? Por último, ¿Cómo se articulan y dialogan las antropologías latinoamericanas con las de otros continentes para repensar nuevos acuerdos sociales?

Recibido: 01 de junio de 2021

Aprobado: 09 de agosto de 2021 


\section{Bibliografía}

ACOSTA, Carla. 2011. Rol que juegan las intérpretes de lengua de señas en la relación de personas sordas - personas oyentes. Master's Diss., Quito, Flacso Ecuador.

ANDRADE, Xavier. 1992. Atrás de los perversos: para una crítica antropológica de las drogas. Licenciatura en antropología, Quito, PUCE.

BARBA, Daniela. 2020. Configuración del campo disciplinar de la antropología sociocultural alrededor de su profesionalización en el Ecuador (1940 - 1972). Licenciatura en antropología, Quito, PUCE.

BOSSANO, Fernanda. 2019. Identidad y lengua de señas ecuatoriana: una etnografía al interior de la comunidad sorda de Quito. Licenciatura en antropología, Quito, PUCE.

BUSTAMENTE, Teodoro; CARRIÓN, Sofía. 2021. “50 años de antropología en Ecuador”. Manuscrito no publicado, Laboratorio de Antropología, PUCE, Quito.

CARRILLO, María. 1995. El sabor de la tradición, escenarios y actores de la cocina tradicional en el centro histórico de Quito. Licenciatura en antropología, Quito, PUCE.

ENSIGNIA, Marco. 1993. Entre el spray y la pared. Graffiti: liminalidad en el espacio urbano. Master's Diss., Quito, Flacso Ecuador.

ESCOBAR, Martha. 1988. La frontera imprecisa: relación de lo natural y lo sagrado en la cultura negra del norte de Esmeraldas. Licenciatura en antropología, Quito, PUCE.

GARCÍA, Fernando. 2011. "La construcción del pensamiento antropológico ecuatoriano: Derroteros y perspectiva”. Alteridades, 21(41): 61-68.

GARCÍA, Fernando. 2014. "La relación entre la antropología mexicana y ecuatoriana: ¿Un camino de ida y vuelta?” Revista Antropologías del Sur, 1: 105-18.

GÓMEZ, Eloy. 2020. "Presentación. La construcción de la antropología en los países andinos”. Gazeta de Antropología, 36(2): 1-11.

HARPER, Douglas. 2002. "Talking about pictures: a case for photo elicitation". Visual Studies, 17(1): 13-26. https://doi.org/10.1080/14725860220137345.

IDROVO, Israel. 2014. Invisibilidad y no videncia: la experiencia urbana de personas ciegas en la ciudad de Cuenca. Master's Diss., Quito, Flacso Ecuador.

JIMENO, Myriam. 2007. "NACIONCENTRISMO: tensiones y configuración de estilos en la antropología sociocultural colombiana”. Revista Colombiana de Antropología, 43: 9-32.

KROTZ, Esteban. 2007. "Cuatro cuestiones cruciales para el desarrollo de nuestras antropologías". En: Angela Giglia, Carlos Grama, y Ana Paula de Teresa (ed.), ¿Adónde va la Antropología? México: Universidad Autónoma Metropolitana, Juan Pablos, Tecnigraf. pp. 157-76.

MARTÍNEZ, Carmen. 2008. "Ecuador: Militants, Priests, Technocrats, and Scholars". En: Deborah Poole (ed.), A companion to Latin American anthropology. Oxford: Blackwell Publishing. pp. 90-108

MONTELLANO, Violeta. 2011. Fotografía realizada por personas con 'ceguera/baja visión' en Quito, Ecuador: oculocentrismo y visualidad alterna. Master's Diss., Quito: Flacso Ecuador.

MORENO, Segundo. 2006. "Estudio introductorio”. En: Pensamiento antropológico ecuatoriano. Quito: Ediciones Banco Central del Ecuador, Corporación Editoria Nacional. pp. 9-130

MURILLO, Rodrigo. 1992. Minería en el altiplano orense: génesis cultural y conflictos. Licenciatura en antropología, Quito, PUCE.

PÉREZ, Rocío. 2020. "La construcción del sujeto antropológico en Ecuador y Bolivia. De una otredad indígena a una otredad plural y descentrada". Gazeta de Antropología, 36(2): 1-15.

PICO, Iris. 2014. Aportes antropológicos para la inclusión laboral de las personas con necesidades especiales. Licenciatura en antropología, Quito, UPS. 
PIÑEIRO, Eleder. 2020. "Antropología en Ecuador. Contexto, relaciones y objetos de estudio". Gazeta de Antropología, 36(2): 1-15.

POESCHEL, Úrsula. 1985. La mujer salasaca: su situación en una época de reestructuración económico - cultural. Licenciatura en antropología, Quito, PUCE.

PRIETO, Mercedes. 2007. “Aportes de la antropología a los estudios de género: Notas para una reflexión”. En: Fernando García (ed.), II Congreso Ecuatoriano de Antropología y Arqueología. Balance de la última década: aportes, retos y nuevos temas. Quito: Abya - Yala. pp. 107-21

PRIETO, Mercedes. 2010. "Indigenismo: La red interamericana". En: Ecuador y México. Vínculo histórico e interculturalidad (1820 - 1970). Quito: Museo de la Ciudad. pp. 250-65.

PRIETO, Mercedes; PÁEZ, Carolina. 2017. "La Misión Andina en Ecuador: doble delegación femenina y sentidos de estado". En: Mercedes Prieto (ed.), El Programa Indigenista Andino, 1951-1973. Las mujeres en los ensambles estatales del desarrollo. Quito: Flacso-Sede Ecuador; Instituto de Estudios Peruanos. pp. 111-62.

REBOLLEDO, Loreto. 1985. El caso de la televisión en Quito; ciudad, cultura y comunicación: una interpretación antropológica. Licenciatura en antropología, Quito, PUCE.

REYGADAS, Luis. 2007. "La desigualdad después del (multi)culturalismo". En: Angela Giglia, Carlos Grama, y Ana Paula De Teresa (ed.), ¿Adónde va la Antropología? México: Universidad Autónoma Metropolitana, Juan Pablos, Tecnigraf. pp. 341-64.

ROJAS, Sonia. 2011. Escuela y discapacidad: representaciones sociales y prácticas de diferencia en la escuela. Master's diss., Quito, Flacso Ecuador.

VIVIENNE, Sonja; BURGESS, Jean. 2013. "The remediation of the personal photograph and the politics of self-representation". Journal of Material Culture, 18(3): 279-98. https://doi.org/10.1177/1359183513492080.

Fernando García

Coordinador de Vinculación con la Sociedad

FLACSO Ecuador

https://orcid.org/0000-0002-9953-045

E-mail: fgarcia@flacso.edu.ec

\section{Carolina Páez}

Profesora, Carrera de Antropología, Facultad de Ciencias Humanas

Pontificia Universidad Católica del Ecuador

https://orcid.org/0000-0003-0947-9073

E-mail: cpaez545@puce.edu.ec 